https://doi.org/10.15407/dopovidi2021.05.003

удК 517.958:512.816

О.В. Локазюк, https://orcid.org/0000-0001-9663-251X

Інститут математики НАН України, Київ

E-mail: sasha.lokazuik@gmail.com

\title{
Ліївські симетрії лінійних систем двох звичайних диференціальних рівнянь другого порядку
}

Представлено членом-кореспондентом НАН Украйни А.Г. Нікітіним

Розв'язано задачу повної групової класифікації класу нормальних лінійних систем звичайних диференціальних рівнянь другого порядку з двома залежними змінними над дійсним полем. Доведення суттєво використовує опис допустимих перетворень цього класу та теорему Лі про реалізацї алгебр Лі на прямій.

Ключові слова: лінійні системи звичайних диференціальних рівнянь, ліївські симетрій, алгебрайчний метод групової класифікації, група еквівалентності.

Опис трансформаційних властивостей і класифікація ліївських симетрій - класичні задачі групового аналізу звичайних диференціальних рівнянь (див. огляди в роботах [1, 2]), хоча тут є багато відкритих проблем, особливо щодо систем звичайних диференціальних рівнянь, зокрема, і лінійних. Важливим класом таких систем є клас $\overline{\mathcal{L}}$ нормальних лінійних систем з $n$ звичайних диференціальних рівнянь другого порядку

$$
\mathbf{x}_{t t}=A(t) \mathbf{x}_{t}+B(t) \mathbf{x}+\mathbf{f}(t)
$$

на $n$ невідомих функцій $x^{1}, \ldots, x^{n}, \mathbf{x}(t)=\left(x^{1}(t), \ldots, x^{n}(t)\right)^{\mathrm{T}}, n \geqslant 2$. Набір $\theta=(A, B, \mathbf{f})$ довільних елементів класу $\overline{\mathcal{L}}$ утворюють довільні гладкі $n \times n$ матричнозначні функції $A$ та $B$ змінної $t$ і довільна гладка векторнозначна функція $\mathbf{f}$ змінної $t$. У літературі відомі лише окремі результати щодо симетрійних та трансформаційних властивостей систем 3 класу $\overline{\mathcal{L}}$. Ліївські симетрії систем із класу $\overline{\mathcal{L}}$ з $A=0$ та сталою матрицею $B$ при $n \leqslant 6$ розглянуто в серії робіт С. Вафа Соха [3], С.В. Мелешка [4], Р. Компоамор-Стурсберга [5, 6]. Задачу групової класифікації таких систем у випадку довільного $n \geqslant 2$ вичерпно розв'язано в [7]. Весь клас $\overline{\mathcal{L}}$, включно із системами з несталими значеннями матриць довільних елементів $A$ та $B$, розглядали з точки зору ліївських симетрій лише для $n=2$ в [8-10] і для $n=3$ в [11].

Ц и т у в а н н я: Локазюк О.В. Ліївські симетрії лінійних систем двох звичайних диференціальних рівнянь другого порядку. Допов. Нащ. акад. наук Укр. 2021. № 5. С. 3-11. https://doi.org/10.15407/dopovidi2021.05.003 
Як приклад реалізації запропонованого в [2] підходу в цій статті виконано повну групову класифікацію класу $\overline{\mathcal{L}}$ у випадку двох залежних змінних над дійсним полем.

Очевидно, що звичайна група еквівалентності $G_{\overline{\mathcal{L}}}^{\sim}$ класу $\overline{\mathcal{L}}$ містить підгрупу, породжену перетвореннями

$$
\begin{aligned}
& \tilde{t}=t, \quad \tilde{\mathbf{x}}=H(t) \mathbf{x}+\mathbf{h}(t), \\
& \tilde{A}=\left(H A+2 H_{t}\right) H^{-1}, \quad \tilde{B}=\left(H B-\tilde{A} H_{t}+H_{t t}\right) H^{-1}, \quad \mathbf{f}=H \mathbf{f}+\mathbf{h}_{t t}-\tilde{A} \mathbf{h}_{t}-\tilde{B} \mathbf{h},
\end{aligned}
$$

де $H$ - довільна гладка невироджена $n \times n$ матричнозначна функція змінної $t$, a $\mathbf{h}-$ довільна гладка векторнозначна функція змінної $t$. Будь-яку систему $\bar{L}_{\theta} 3$ класу $\overline{\mathcal{L}}$ можна відобразити за допомогою перетворення вигляду (2), де $H$ задовольняє матричне рівняння $H_{t}+1 / 2 H A=0$, a $\mathbf{h}-$ частинний розв'язок системи $\bar{L}_{\theta}$, у систему $\bar{L}_{\tilde{\theta}} 3$ того ж класу з $\tilde{A}=0, \tilde{B}=H\left(B-1 / 2 A_{t}+1 / 4 A^{2}\right) H^{-1}$ та $\mathbf{f}=\mathbf{0}$. Таким чином, задачу групової класифікації класу $\overline{\mathcal{L}}$ зводимо до задачі групової класифікації підкласу $\mathcal{L}^{\prime}$, який виокремлено зв'язками $A=0$ та $\mathbf{f}=\mathbf{0}$. Після тривіальної репараметризизації класу $\mathcal{L}^{\prime}$ через виключення $A$ та $\mathbf{f}$ з набору довільних елементів цього класу і перепозначення $B$ як $V$ системи з класу $\mathcal{L}^{\prime}$ набувають вигляду

$$
\mathbf{x}_{t t}=V(t) \mathbf{x},
$$

де $V$ - довільна гладка $n \times n$ матричнозначна функція змінної $t, V=V(t)=\left(V^{a b}(t)\right)_{a, b=1}^{n}$. Далі $L_{V}^{\prime}$ - система з класу $\mathcal{L}^{\prime}$, яка відповідає фіксованому значенню матричнозначної параметр-функції $V$.

Розглянемо підклас $\mathcal{L}_{0}^{\prime}$ класу $\mathcal{L}^{\prime}$, в який входять системи $L_{V}^{\prime}$ з $V(t)=v(t) E, t \in \mathcal{I}$, де $v$ пробігає множину функцій змінної $t$, тобто $\{V(t) \mid t \in \mathcal{I}\} \subseteq\langle E\rangle$. Тут і далі $E$ - одинична $n \times n$ матриця. Для таких $V$ можна казати, що $V$ пропорційна $E$ із залежним від часу коефіцієнтом пропорційності. Іншими словами, підклас $\mathcal{L}_{0}^{\prime}$, який є сингулярним у класі $\mathcal{L}^{\prime}$, виокремлено з класу $\mathcal{L}^{\prime}$ алгебраїчними рівняннями

$$
V^{a b}=0, \quad a \neq b, \quad V^{11}=\cdots=V^{n n} .
$$

Доповненням сингулярного підкласу $\mathcal{L}_{0}^{\prime}$ в $\mathcal{L}^{\prime}$ є регулярний підклас $\mathcal{L}_{1}^{\prime}:=\mathcal{L}^{\prime} \backslash \mathcal{L}_{0}^{\prime}$. Таким чином, $\mathcal{L}^{\prime}=\mathcal{L}_{0}^{\prime} \sqcup \mathcal{L}_{1}^{\prime}$.

Теорема 1 ([2, теорема 4]). Групу еквівалентності $G_{\mathcal{L}^{\prime}}^{\sim}$ класу $\mathcal{L}^{\prime}$ складають перетворення вигляду

$$
\begin{aligned}
& \tilde{t}=T(t), \quad \tilde{\mathbf{x}}=\left|T_{t}\right|^{1 / 2} C \mathbf{x}, \\
& \tilde{V}=\frac{1}{T_{t}{ }^{2}} C V C^{-1}+\frac{2 T_{t} T_{t t t}-3 T_{t t}{ }^{2}}{4 T_{t}{ }^{4}} E,
\end{aligned}
$$

де $T=T(t)-$ довільна функція змінної $t, T_{t} \neq 0, C-$ довільна стала невироджена $n \times n$ матриия. Група еквівалентності підкласу $\mathcal{L}_{1}^{\prime}$ та канонічна істотна група еквівалентності підкласу $\mathcal{L}_{0}^{\prime}$ збігаються з групою $G_{\mathcal{L}^{\prime}}^{\sim}$ 
За допомогою перетворень еквівалентності класу $\mathcal{L}^{\prime}$ довільну систему із сингулярного підкласу $\mathcal{L}_{0}^{\prime}$ можна звести до елементарної системи $\mathbf{x}_{t t}=\mathbf{0}$, алгебра інваріантності

$$
\mathfrak{g}_{0}=\left\langle\partial_{t}, \partial_{x^{a}}, t \partial_{t}, x^{a} \partial_{t}, t \partial_{x^{a}}, x^{a} \partial_{x^{b}}, t x^{a} \partial_{t}+x^{a} x^{c} \partial_{x^{c}}, t^{2} \partial_{t}+t x^{c} \partial_{x^{c}}\right\rangle
$$

якої ізоморфна алгебрі $\mathfrak{s l}(n+2, \mathbb{R})$ [12], а їі розмірність дорівнює $(n+2)^{2}-1$.

Таким чином, задача групової класифікації класу $\mathcal{L}^{\prime}$ зводиться до задачі групової класифікації його регулярного підкласу $\mathcal{L}_{1}^{\prime}$.

Наслідок 2. Максимальну алгебру лївської інваріантності $\mathfrak{g}_{V}$ системи $L_{V}^{\prime}$ iз класу $\mathcal{L}_{1}^{\prime}$ утворюють векторні поля вигляду

$$
Q=\tau \partial_{t}+\left(1 / 2 \tau_{t} x^{a}+\Gamma^{a b} x^{b}+\chi^{a}\right) \partial_{x^{a}},
$$

де векторнозначна функція $\chi(t)=\left(\chi^{1}(t), \ldots, \chi^{n}(t)\right)^{\mathrm{T}}-$ довільний розв'язок системи $L_{V}^{\prime}$, $\tau(t)$ - довільна гладка функція, Г - довільна стала $n \times n$ матрищя, які задовольняють класифікаційне рівняння

$$
\tau V_{t}=[\Gamma, V]-2 \tau_{t} V+1 / 2 \tau_{t t t} E
$$

Алгебра $\mathfrak{g}_{V} \in$ напівпрямою сумою $\mathfrak{g}_{V}=\mathfrak{g}_{V}^{\text {ess }} \Subset \mathfrak{g}_{V}^{\text {lin }}$, де $\mathfrak{g}_{V}^{\text {lin }}-$ абелевий ідеал, який пов’язаний з лінійною суперпозицією розв'язків, а $\mathfrak{g}_{V}^{\text {ess }}$ - його доповняльна підалгебра, яку називають суттєвою алгеброю ліївської інваріантності системи $L_{V}^{\prime}$. Алгебра $\mathfrak{g}_{V}^{\text {ess }}$ обов'язково містить підалгебру $\mathfrak{s}_{Q}:=\left\{Q_{\Gamma}:=\Gamma^{a b} x^{b} \partial_{x^{a}} \mid \Gamma \in \mathfrak{s}\right\}$, де $\mathfrak{s}-$ підалгебра $\mathfrak{s l}(2, \mathbb{R})$, подвійний централізатор якої збігається з нею, тобто $\mathrm{C}_{\mathfrak{s l}(2, \mathbb{R})}\left(\mathrm{C}_{\mathfrak{s l}(2, \mathbb{R})}(\mathfrak{s})\right)=\mathfrak{s}$. Базис алгебри $\mathfrak{g}_{V}^{\text {ess }}$ вичерпують:

- векторне поле $I=x^{a} \partial_{x}$, що породжує ядро ліївської алгебри інваріантності $\mathfrak{g}_{\mathcal{L}^{\prime}}$,

- $p$ векторних полів $\mathscr{Q}_{s}=\Gamma_{s}^{a b} x^{b} \partial_{x^{a}}, s=1, \ldots, p$, де матриці $\Gamma_{s}=\left(\Gamma_{s}^{a b}\right)$ утворюють базис підалгебри $\mathfrak{s}=\mathfrak{s}_{V}$ алгебри $\mathfrak{s l}(n, \mathbb{R}), 0 \leqslant p:=\operatorname{dim} \mathfrak{s} \leqslant \operatorname{dim} \mathfrak{s l}(n, \mathbb{R})=n^{2}-1$,

- $k$ векторних полів $Q_{p+1}=\tau^{l} \partial_{t}+\left(1 / 2 \tau_{t}^{l} x^{a}+\Gamma_{p+\mathrm{l}}^{a b} x^{b}\right) \partial_{x^{a}}$ з лінійно незалежними $t$-компонентами $\tau^{\imath}, \mathbf{\imath}=1, \ldots, k$, де $k \in\{0,1,2,3\}$.

Іншими словами, алгебру $\mathfrak{g}_{V}^{\text {ess }}$ можна представити як $\mathfrak{g}_{V}^{\text {ess }}=\mathfrak{i} \oplus\left(\mathfrak{t} \oplus \mathfrak{s}^{\text {vf }}\right)$, де $\mathfrak{i}:=\langle I\rangle-$ ідеал алгебри $\mathfrak{g}_{V}^{\mathrm{ess}}$, що є спільним для всіх систем $L_{V}^{\prime} 3$ класу $\mathcal{L}_{1}^{\prime}, \mathfrak{s}^{\mathrm{vf}}=\mathfrak{s}_{V}^{\mathrm{vf}}:=\left\langle Q_{s}, s=1, \ldots, p\right\rangle=$ $=\left\{\Gamma^{a b} x^{b} \partial_{x^{a}} \mid \Gamma \in \mathfrak{s}\right\}-$ ідеал алгебри $\mathfrak{g}_{V}^{\text {ess }}, \mathfrak{t}=\mathfrak{t}_{V}:=\left\langle Q_{p+\mathfrak{l}}, \mathfrak{l}=1, \ldots, k\right\rangle-$ підалгебра алгебри $\mathfrak{g}_{V}^{\text {ess }}$. Більше того, $k:=\operatorname{dim} \mathfrak{t} \in\{0,1,2\}$ та $p:=\operatorname{dim} \mathfrak{s}^{\mathrm{vf}} \leqslant n^{2}-2 n+1$ (див. доведення в [2]).

Спираючись на результати роботи [2], виконаємо повну групову класифікацію нормальних лінійних систем двох звичайних диференціальних рівнянь другого порядку, тобто систем $L_{V}^{\prime}$ з класу $\mathcal{L}_{1}^{\prime}$ вигляду

$$
\mathbf{x}_{t t}=V(t) \mathbf{x}, \quad V(t)=\left(\begin{array}{ll}
V^{11}(t) & V^{12}(t) \\
V^{21}(t) & V^{22}(t)
\end{array}\right),
$$

де $\mathbf{x}=\left(x^{1}, x^{2}\right)$, набір довільних елементів $V$ пробігає множину гладких $2 \times 2$ матричнозначних функцій змінної $t$, які непропорційні одиничній матриці $E$. 
Виберемо як базис алгебри $\mathfrak{s l}(2, \mathbb{R})$ такий набір матриць:

$$
S_{1}=\left(\begin{array}{cc}
0 & 1 \\
0 & 0
\end{array}\right), \quad S_{2}=\left(\begin{array}{cc}
1 & 0 \\
0 & -1
\end{array}\right), \quad S_{3}=\left(\begin{array}{cc}
0 & 0 \\
-1 & 0
\end{array}\right),
$$

які задовольняють комутаційні співвідношення

$$
\left[S_{1}, S_{2}\right]=-2 S_{1}, \quad\left[S_{2}, S_{3}\right]=-2 S_{3}, \quad\left[S_{1}, S_{3}\right]=-S_{2} .
$$

Повний список $\mathrm{SL}(2, \mathbb{R})$-нееквівалентних підалгебр $\mathfrak{s}$ алгебри $\mathfrak{s t}(2, \mathbb{R})$ вичерпують такі підалгебри: $\{0\},\left\langle S_{1}\right\rangle,\left\langle S_{2}\right\rangle,\left\langle S_{1}+S_{3}\right\rangle,\left\langle S_{1}, S_{2}\right\rangle$ та $\mathfrak{s l}(2, \mathbb{R})$. Подвійні централізатори $\mathrm{C}_{\mathfrak{s l}(2, \mathbb{R})}\left(\mathrm{C}_{\mathfrak{s l}(2, \mathbb{R})}(\mathfrak{s})\right)$ цих підалгебр відповідно дорівнюють $\{0\},\left\langle S_{1}\right\rangle,\left\langle S_{2}\right\rangle,\left\langle S_{1}+S_{3}\right\rangle$, $\mathfrak{s l}(2, \mathbb{R})$ та $\mathfrak{s l}(2, \mathbb{R})$. Оскільки $\mathrm{C}_{\mathfrak{s l}(2, \mathbb{R})}\left(\mathrm{C}_{\mathfrak{s l}(2, \mathbb{R})}(\mathfrak{s})\right) \neq \mathfrak{s}$ тоді і лише тоді, коли $\mathfrak{s}=\left\langle S_{1}, S_{2}\right\rangle$, то це єдина підалгебра алгебри $\mathfrak{s l}(2, \mathbb{R})$, яка не підходить для групової класифікації класу $\mathcal{L}_{1}^{\prime}$ при $n=2$. Підалгебра $\mathfrak{s}=\mathfrak{s l}(2, \mathbb{R})$ також не придатна для цієї класифікації, оскільки будьяка система, яка допускає відповідну алгебру $\mathfrak{s}^{\mathrm{vf}}$, належить до сингулярного підкласу.

Теорема 3. Повний список $G_{\mathcal{L}^{\prime}}^{\sim}$-нееквівалентних розширень суттєвих лї̈вських симетрій у класі $\mathcal{L}_{1}^{\prime}$ з $n=2$ вичерпують випадки:

0. Загальний випадок $V(t): \mathfrak{g}_{V}^{\text {ess }}=\langle I\rangle$;

1. $V=v(t) S_{1}: \mathfrak{g}_{V}^{\text {ess }}=\left\langle I, x^{2} \partial_{x^{1}}\right\rangle$;

2. $V=v(t)\left(S_{1}+S_{3}\right): \mathfrak{g}_{V}^{\text {ess }}=\left\langle I, x^{1} \partial_{x^{2}}-x^{2} \partial_{x^{1}}\right\rangle$;

3. $V=v(t) S_{2}: \mathfrak{g}_{V}^{\text {ess }}=\left\langle I, x^{1} \partial_{x^{1}}-x^{2} \partial_{x^{2}}\right\rangle$;

4. $V=\varepsilon E+\left(\beta_{1}-2 \beta_{2} t+\beta_{3} t^{2}\right) S_{1}+\left(\beta_{2}-\beta_{3} t\right) S_{2}+\beta_{3} S_{3},\left(\beta_{2}, \beta_{3}\right) \neq(0,0): \mathfrak{g}_{V}^{\text {ess }}=\left\langle I, \partial_{t}+x^{2} \partial_{x^{1}}\right\rangle ;$

5. $V=\varepsilon E+\mu\left(S_{1}+S_{3}\right)+v \cos (2 t)\left(S_{1}-S_{3}\right)+v \sin (2 t) S_{2}, \quad v \neq 0: \mathfrak{g}_{V}^{\text {ess }}=\left\langle I, \partial_{t}+x^{2} \partial_{x^{1}}-x^{1} \partial_{x^{2}}\right\rangle$;

6. $V=\varepsilon E+\beta_{1} \mathrm{e}^{2 t} S_{1}+\beta_{2} S_{2}+\beta_{3} \mathrm{e}^{-2 t} S_{3},\left(\beta_{1} \beta_{2}, \beta_{2} \beta_{3}, \beta_{3} \beta_{1}\right) \neq(0,0,0)$ :

$$
\mathfrak{g}_{V}^{\text {ess }}=\left\langle I, \partial_{t}+x^{1} \partial_{x^{1}}-x^{2} \partial_{x^{2}}\right\rangle
$$

7. $V=\varepsilon E+S_{1}+S_{3}: \mathfrak{g}_{V}^{\text {ess }}=\left\langle I, x^{1} \partial_{x^{2}}-x^{2} \partial_{x^{1}}, \partial_{t}\right\rangle$;

8. $V=\varepsilon E+\mathrm{e}^{2 \gamma t} S_{1}, 4 \varepsilon \neq \gamma^{2}: \mathfrak{g}_{V}^{\text {ess }}=\left\langle I, x^{2} \partial_{x^{1}}, \partial_{t}+\gamma\left(x^{1} \partial_{x^{1}}-x^{2} \partial_{x^{2}}\right)\right\rangle$;

9. $V=\varepsilon E+S_{2}: \mathfrak{g}_{V}^{\text {ess }}=\left\langle I, x^{1} \partial_{x^{1}}-x^{2} \partial_{x^{2}}, \partial_{t}\right\rangle$;

10. $V=S_{1}: \mathfrak{g}_{V}^{\mathrm{ess}}=\left\langle I, x^{2} \partial_{x^{1}}, \partial_{t}, t \partial_{t}+2 x^{1} \partial_{x^{1}}\right\rangle$.

Tym $\varepsilon, \gamma, \mu, v, \beta_{1}, \beta_{2}, \beta_{3} \in \mathbb{R}, I:=x^{1} \partial_{x^{1}}+x^{2} \partial_{x^{2}}, v=v(t)-$ довільна гладка функція з $\tau v_{t} \neq$ $\neq\left(\kappa-2 \tau_{t}\right) \nu$ для будь-якої сталої $\kappa \in \mathbb{R}$ та будь-якої функції $\tau=\tau(t)$ з $\tau_{t t t}=0.3$ точністю до $G_{\mathcal{L}^{\prime}}^{\sim}$ еквівалентності можна вважати, що $V$ - ненульова матричнозначна функція змінної $t$ з нульовим слідом у випадку $0, \beta_{2}=0$ при $\beta_{3} \neq 0$ або $\beta_{1}=0$ при $\beta_{3}=0$ та $\beta_{2} \neq 0$ у випадку 4, одне з ненульових $\beta_{1}$ або $\beta_{3}$ дорівнюе 1 у випадку $6, \gamma \in\{0,1\}$ у випадку 8 та $\vee>0$ у випадку 5.

Доведення. 3 класифікаційної умови (7) випливає, що $I \in \mathfrak{g}_{V}^{\text {ess }}$ для будь-якої системи $L_{V}^{\prime}$ з класу $\mathcal{L}_{1}^{\prime}$, причому $\mathfrak{g}_{V}^{\text {ess }}=\langle I\rangle$ для загальної системи цього класу (випадок 0 теореми).

Далі розглянемо розширення суттєвих ліївських симетрій у класі $\mathcal{L}_{1}^{\prime}$ для можливих значень розмірності підалгебри $\mathfrak{t}$, тобто $k=0, k=1$ та $k=2$.

$k=0.3$ самого початку з точністю до $G_{\mathcal{L}^{\prime}}^{\sim}$-еквівалентності зручно покласти $\operatorname{tr} V=0$. Підалгебра $\mathfrak{s}=\{0\}$ алгебри $\mathfrak{s t}(2, \mathbb{R})$ не підходить, оскільки вона відповідає випадку загальної 
матричнозначної функції $V(t)$. Підалгебри $\left\langle S_{1}\right\rangle,\left\langle S_{1}+S_{3}\right\rangle$ та $\left\langle S_{2}\right\rangle$ приводять відповідно до випадків 1, 2 та 3 теореми, де $V=v(t) \Gamma, \mathfrak{g}_{V}^{\text {ess }}=\left\langle I, Q_{\Gamma}\right\rangle$ з матрицею $\Gamma$, рівною відповідно $S_{1}$, $S_{1}+S_{3}$ та $S_{2}$. Подальші розширення ліївських симетрій можливі тоді і лише тоді, коли $\tau v_{t}=\left(\kappa-2 \tau_{t}\right) v$ для деякої сталої $\kappa$ та деякої функції $\tau$ від $t$, що задовольняє умову $\tau_{t t t}=0$, тобто для $k=0$ параметр-функція $v$ не має задовольняти жодне з рівнянь такого вигляду.

$k=1$. Тоді з точністю до $G_{\mathcal{L}^{\prime}}^{\sim}$-еквівалентності $\mathfrak{g}_{V}^{\mathrm{ess}}=\langle I\rangle \oplus\left(\langle P\rangle \oplus \mathfrak{s}^{\mathrm{vf}}\right)$, де $P:=\partial_{t}+\Upsilon^{a b} x^{b} \partial_{x^{a}}$. Матричнозначна параметр-функція $V$ має вигляд [2]

$$
V(t)=\varepsilon E+\mathrm{e}^{t \Upsilon} W \mathrm{e}^{-t \Upsilon}=\varepsilon E+\sum_{l=0}^{\infty} \frac{t^{l}}{l !} K_{l}, \quad K_{0}:=W, \quad K_{l}:=\left[\Upsilon, K_{l-1}\right], \quad l=1,2, \ldots
$$

з $W \neq 0$. Далі як кандидати для підалгебр $\mathfrak{s}$ окремо розглянемо кожен з придатних елементів повного списку $\operatorname{SL}(2, \mathbb{R})$-нееквівалентних підалгебр алгебри $\mathfrak{s l}(2, \mathbb{R})$, а саме $\{0\}$, $\left\langle S_{1}\right\rangle,\left\langle S_{2}\right\rangle$ та $\left\langle S_{1}+S_{3}\right\rangle$.

Підалгебра $\mathfrak{s}=\{0\}$ не приводить до значних попередніх обмежень щодо матриць $\Upsilon$ та $W$. Лише очевидно, що матриця $\Upsilon$ також ненульова, оскільки інакше $\mathrm{C}_{\mathfrak{s l}(2, \mathbb{R})}\left(\left\{K_{l}, l \in \mathbb{N}_{0}\right\}\right)=$ $=\langle W\rangle \neq\{0\}=\mathfrak{s}$. Таким чином, розгляд у цьому випадку зводиться до класифікації пар ненульових $2 \times 2$ матриць 3 точністю до перетворень подібності. Фіксуємо кожну з можливих $2 \times 2$ жорданових нормальних форм як значення для параметр-матриці $\Upsilon: \Upsilon=S_{1}, \Upsilon=\gamma S_{2}$ та $\Upsilon=\gamma\left(S_{1}+S_{3}\right)$, де $\gamma \neq 0$, а тому внаслідок масштабування змінної $t$ завжди можна вважати, що $\gamma=1$. Для кожної з цих матриць $\Upsilon$ маємо $\mathrm{C}_{\mathfrak{s l}(2, \mathbb{R})}(\{\Upsilon\})=\langle\Upsilon\rangle$. Отже, будь-яка невироджена матриця $M$, яка комутує з фіксованим виглядом матриці $\Upsilon$, пропорційна $\mathrm{e}^{\beta \Upsilon}$ для деякого $\beta \in \mathbb{R}$. Відповідно до [2, леми 27] матрицю $W$ можна звести до вигляду $\tilde{W}=\mathrm{e}^{\beta \Upsilon} W \mathrm{e}^{-\beta \Upsilon}$, зберігаючи при цьому вигляд матриці $\Upsilon$. Нехай матриця $W$ має загальний вигляд, тобто $W=: K_{0}=\beta_{1} S_{1}+\beta_{2} S_{2}+\beta_{3} S_{3}$ із $\left(\beta_{1}, \beta_{2}, \beta_{3}\right) \neq(0,0,0)$. Розглянемо всі можливі спрощення вигляду матриці $W$ за допомогою зазначених перетворень.

Якщо $\Upsilon=S_{1}$, то

$$
K_{1}=-2 \beta_{2} S_{1}-\beta_{3} S_{2}, K_{2}=2 \beta_{3} S_{1}, K_{l}=0, l \geqslant 3,
$$

і централізатор $\mathrm{C}_{\mathfrak{s l}(2, \mathbb{R})}\left(\left\{K_{0}, K_{1}, K_{2}\right\}\right)$ збігається з $\mathfrak{s}=\{0\}$ тоді і лише тоді, коли $\left(\beta_{2}, \beta_{3}\right) \neq(0,0)$. 3 точністю до $G_{\mathcal{L}^{\prime}}^{\sim}$-еквівалентності можна покласти $\beta_{2}=0$, якщо $\beta_{3} \neq 0$, або $\beta_{1}=0$, якщо $\beta_{3}=0$ та $\beta_{2} \neq 0$. У результаті отримаємо випадок 4 теореми.

Якщо $\Upsilon=S_{2}$, то

$$
K_{l}=\left[\Upsilon, K_{l-1}\right]=2^{l} \beta_{1} S_{1}+(-2)^{l} \beta_{3} S_{3}, \quad l \in \mathbb{N},
$$

а централізатор $\mathrm{C}_{\mathfrak{s l}(2, \mathbb{R})}\left(\left\{K_{l}, l \in \mathbb{N}_{0}\right\}\right)$, збігається $3 \mathfrak{s}=\{0\}$ тоді і лише тоді, коли щонайменше дві зі сталих $\beta_{1}, \beta_{2}$ та $\beta_{3}$ є ненульовими. Одне із ненульових значень $\beta_{1}$ чи $\beta_{3}$ можна покласти рівним 1 , використовуючи зсув за змінною $t$. Таким чином, отримаємо випадок 6 теореми.

Якщо $\Upsilon=S_{1}+S_{3}$, то можна покласти $\beta_{2}=0$, тобто $W=: K_{0}=\beta_{1} S_{1}+\beta_{3} S_{3}, \quad K_{2 l+1}=$ $=\left(\beta_{1}-\beta_{3}\right)(-4)^{l} S_{2}, K_{2 l+2}=2\left(\beta_{1}-\beta_{3}\right)(-4)^{l}\left(S_{3}-S_{1}\right), l \in \mathbb{N}_{0}$, а тому $\mathrm{C}_{\mathfrak{s l}(2, \mathbb{R})}\left(\left\{K_{l}, l \in \mathbb{N}_{0}\right\}\right)=\{0\}=\mathfrak{s}$ тоді і лише тоді, коли $\beta_{1}-\beta_{3} \neq 0.3$ точністю до зсуву за змінною $t$, кратному $\pi / 2$, мож- 
на вважати, що $\beta_{1}-\beta_{3}>0$. Якщо ввести позначення $\mu:=\left(\beta_{1}+\beta_{3}\right) / 2$ та $v:=\left(\beta_{1}-\beta_{3}\right) / 2$, то приходимо до випадку 5 теореми.

Надалі s позначає доповняльний підпростір підалгебри $\mathfrak{s}$ у нормалізаторі $\mathrm{N}_{\mathfrak{s l}(n, \mathbb{R})}(\mathfrak{s})$.

Для підалгебри $\mathfrak{s}=\left\langle S_{1}\right\rangle$ маємо $\mathrm{N}_{\mathfrak{s l}(n, \mathbb{R})}(\mathfrak{s})=\left\langle S_{1}, S_{2}\right\rangle$ і виберемо $\mathrm{s}=\left\langle S_{2}\right\rangle$, а $\Upsilon \in\left\langle S_{2}\right\rangle$, тобто $\Upsilon=\gamma S_{2}$ з $\gamma \in \mathbb{R}$. Більш того, $W \in \mathrm{C}_{\mathfrak{s l}(n, \mathbb{R})}(\mathfrak{s})=\left\langle S_{1}\right\rangle$ та $W \neq 0$, звідки $W=\beta S_{1}$ з $\beta \neq 0$, а тому $K_{l}=\beta(2 \gamma)^{l} S_{1}, l \in \mathbb{N}_{0}$. У результаті отримаємо випадок 8 теореми, де $4 \varepsilon \neq \gamma^{2}$ (інакше $k=2$, див. розгляд нижче). Додатково можна покласти $\gamma \in\{0,1\}$ та $\beta=1$ відповідно за допомогою масштабування та зсуву за змінною $t$.

Якщо $\mathfrak{s}=\left\langle S_{2}\right\rangle$, то $\mathrm{N}_{\mathfrak{s l}(2, \mathbb{R})}(\mathfrak{s})=\mathfrak{s}, \mathrm{s}=\{0\}$, а тому єдиний можливий вибір для матриці $\Upsilon-$ це $\Upsilon=0$. Крім того, $K_{0}:=W=\beta S_{2} 3 \beta \neq 0$, оскільки $W \in \mathrm{C}_{\mathfrak{s l}(2, \mathbb{R})}(\mathfrak{s})=\left\langle S_{2}\right\rangle$ та $W \neq 0, K_{l}=0$ для $l \geqslant 1, \mathrm{C}_{\mathfrak{s l}(2, \mathbb{R})}\left(\left\{K_{0}\right\}\right)=\mathfrak{s . ~ М о ж н а ~ п о к л а с т и ~} \beta=1$ за допомогою масштабування змінної $t$ та перестановки $x^{1}$ i $x^{2}$, що приводить до випадку 9 теореми.

Для підалгебри $\mathfrak{s}=\left\langle S_{1}+S_{3}\right\rangle$ нормалізатор $\mathrm{N}_{\mathfrak{s l}(2, \mathbb{R})}(\mathfrak{s})$ також збігається $3 \mathfrak{s}$. Тому $\mathrm{s}=\{0\}$, $\Upsilon=0, W=: K_{0}=\beta\left(S_{1}+S_{3}\right)$ з $\beta \neq 0$, та $K_{l}=0, l \geqslant 1$. За допомогою масштабування змінної $t$ та перестановки $x^{1}$ та $x^{2}$ знову можна покласти $\beta=1$. У результаті отримаємо випадок 7 теореми.

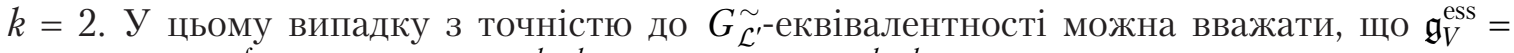
$=\langle I\rangle \oplus\left(\langle P, D\rangle \mp \mathfrak{s}^{\mathrm{vf}}\right)$, де $P:=\partial_{t}+\Upsilon^{a b} x^{b} \partial_{x^{a}}, D:=t \partial_{t}+\Lambda^{a b} x^{b} \partial_{x^{a}}$. Тут і далі відповідні обгрунтування для випадку $k=2$ див. у [2]. Оскільки $W \neq 0$, то матриця $\Lambda$ може мати лише один ланцюжок із двох власних значень, різниця яких дорівнює 2. Більш того, лише різниця власних значень є суттєвою і матрицю $\Lambda$ можна вважати діагоналізовною. Таким чином, можна вибрати матрицю $\Lambda$ у вигляді $\Lambda=\operatorname{diag}(2,0)$. Тоді $\Upsilon=0, W=: K_{0}=S_{1}$ з точністю до $\mathrm{SL}(2, \mathbb{R})$-еквівалентності, $K_{l}=0, l \in \mathbb{N}$, та $\mathrm{C}_{\mathfrak{s l}(2, \mathbb{R})}\left(\left\{K_{0}\right\}\right)=\left\langle S_{1}\right\rangle=\mathfrak{s}$, що приводить до випадку 10 теореми.

Таким чином, теорема 3 дає повний розв’язок задачі групової класифікації для регулярного підкласу $\mathcal{L}_{1}^{\prime}$ класу $\mathcal{L}^{\prime}$. Оскільки будь-яка система з сингулярного підкласу $\mathcal{L}_{0}^{\prime}$ еквівалентна елементарній, це означає повний розв’язок задачі групової класифікації для нормальних лінійних систем двох звичайних диференціальних рівнянь другого порядку. Крім того, теорему можна легко поширити на випадок комплексного поля. Над комплексним полем випадки 2, 5 та 7 теореми відповідно зводяться до випадків 1, 6 та 9.

Теорема 3 покращує й уточнює відомі результати щодо ліївських симетрій нормальних лінійних систем двох звичайних диференціальних рівнянь другого порядку [8-10] i $\epsilon$ ілюстрацією розробленого в роботі [2] методу розв’язання задачі групової класифікації лінійних систем вигляду (1) для довільного $n$.

Прямим наслідком теореми 3 є таке твердження щодо розмірностей алгебр ліївських симетрій нормальних лінійних систем двох звичайних диференціальних рівнянь другого порядку.

\section{Наслідок 4.}

1. $\operatorname{dim} \mathfrak{g}_{V} \in\{5,6,7,8\}$ для будъ-якої системи $L_{V}^{\prime}$ з класу $\mathcal{L}_{1}^{\prime}$.

2. $\operatorname{dim} \mathfrak{g}_{\theta} \in\{5,6,7,8,15\}$ для будь-якої системи $\bar{L}_{\theta}$ з класу $\overline{\mathcal{L}}$.

3. Будь-яка система $\bar{L}_{\theta} \in \overline{\mathcal{L}} 3 \operatorname{dim} \mathfrak{g}_{\theta}>8 \in G_{\overline{\mathcal{L}}}^{\sim}$-еквівалентною елементарній системі $\mathbf{x}_{t t}=\mathbf{0}$. 
4. Будъ-яка система $\bar{L}_{\theta} \in \overline{\mathcal{L}} 3 \operatorname{dim} \mathfrak{g}_{\theta} \geqslant 7 \in G_{\overline{\mathcal{L}}}^{\sim}$-еквівалентною однорідній системі зі сталими матричними коефіцієнтами.

3 використанням перетворень еквівалентності як простий наслідок теореми 3 отримаємо розв'язок задачі групової класифікації для класу $\mathcal{L}_{1}$ регулярних лінійних однорідних систем вигляду (1) для $n=2$ з довільними елементами $\vartheta=(A, B)$.

Наслідок 5. Повний список $G_{\mathcal{L}}^{\sim}$-нееквівалентних розширень суттєвих ліӥвських симетрій у класі $\mathcal{L}_{1}$ з $n=2$ вичерпують такі випадки:

0. Загальний випадок $A=0, B=V(t): \mathfrak{g}_{9}^{\text {ess }}=\langle I\rangle$;

1. $A=0, B=v(t) S_{1}: \mathfrak{g}_{\vartheta}^{\mathrm{ess}}=\left\langle I, x^{2} \partial_{x^{1}}\right\rangle$;

2. $A=0, B=v(t)\left(S_{1}+S_{3}\right): \mathfrak{g}_{\vartheta}^{\text {ess }}=\left\langle I, x^{1} \partial_{x^{2}}-x^{2} \partial_{x^{1}}\right\rangle$;

3. $A=0, B=v(t) S_{2}: \mathfrak{g}_{\vartheta}^{\text {ess }}=\left\langle I, x^{1} \partial_{x^{1}}-x^{2} \partial_{x^{2}}\right\rangle$;

4. $A=-2 S_{1}, B=\varepsilon E+\beta_{1} S_{1}+\beta_{2} S_{2}+\beta_{3} S_{3},\left(\beta_{2}, \beta_{3}\right) \neq(0,0): \mathfrak{g}_{\vartheta}^{\text {ess }}=\left\langle I, \partial_{t}\right\rangle$;

5. $A=-2\left(S_{1}+S_{3}\right), B=\varepsilon E+\beta_{1} S_{1}+\beta_{3} S_{3}, \beta_{1} \neq \beta_{3}: \mathfrak{g}_{9}^{\mathrm{ess}}=\left\langle I, \partial_{t}\right\rangle$;

6. $A=-2 S_{2}, B=\varepsilon E+\beta_{1} S_{1}+\beta_{2} S_{2}+\beta_{3} S_{3},\left(\beta_{1} \beta_{2}, \beta_{2} \beta_{3}, \beta_{3} \beta_{1}\right) \neq(0,0,0): \mathfrak{g}_{\vartheta}^{\text {ess }}=\left\langle I, \partial_{t}\right\rangle$;

7. $A=0, B=\varepsilon E+S_{1}+S_{3}: \mathfrak{g}_{\vartheta}^{\text {ess }}=\left\langle I, x^{1} \partial_{x^{2}}-x^{2} \partial_{x^{1}}, \partial_{t}\right\rangle$;

8. $A=-2 \gamma S_{2}, B=\left(\varepsilon-\gamma^{2}\right) E+S_{1}, 4 \varepsilon \neq \gamma^{2}: \mathfrak{g}_{\vartheta}^{\text {ess }}=\left\langle I, \mathrm{e}^{-2 \gamma t} x^{2} \partial_{x^{1}}, \partial_{t}\right\rangle$;

9. $A=0, B=\varepsilon E+S_{2}: \mathfrak{g}_{\vartheta}^{\mathrm{ess}}=\left\langle I, x^{1} \partial_{x^{1}}-x^{2} \partial_{x^{2}}, \partial_{t}\right\rangle$;

10. $A=0, B=S_{1}: \mathfrak{g}_{\vartheta}^{\mathrm{ess}}=\left\langle I, x^{2} \partial_{x^{1}}, \partial_{t}, t \partial_{t}+2 x^{1} \partial_{x^{1}}\right\rangle$.

Tym $\varepsilon, \gamma, \beta_{1}, \beta_{2}, \beta_{3} \in \mathbb{R}, I:=x^{1} \partial_{x^{1}}+x^{2} \partial_{x^{2}}, v=v(t)-$ довільна гладка функція змінної $t 3$ $\tau v_{t} \neq\left(\kappa-2 \tau_{t}\right)$ ә для будь-якої сталої $\kappa \in \mathbb{R}$ та будь-якоїфункиї $\tau=\tau(t)$ з $\tau_{t t t}=0.3$ точністю до $G_{\mathcal{L}^{\prime}}^{\sim}$-еквівалентності можна вважати, що $V-$ ненульова матричнозначна функція змінної $t$ з нульовим слідом у випадку $0, \beta_{2}=0$ при $\beta_{3} \neq 0$ або $\beta_{1}=0$ при $\beta_{3}=0$ та $\beta_{2} \neq 0$ у випадку 4, одна з ненульових сталих $\beta_{1}$ або $\beta_{3}$ дорівнює 1 у випадку 6,,$\in\{0,1\}$ у випадку 8 та $\beta_{1}>\beta_{3}$ у випадку 5 .

Оскільки класифікація підалгебр алгебри $\mathfrak{s l}(3, \mathbb{R})$ відома [13], то результати роботи [2] можна також використати для повної групової класифікації систем (3) у випадку трьох залежних змінних. Але така класифікація є нетривіальною та громіздкою і вимагає окремого розгляду.

Автор висловлюе щиру вдячність В.М. Бойку та Р.О. Поповичу за постановку задачі, постійну увагу і допомогу, конструктивні зауваження до роботи та плідні дискусї. Дослідження виконувалися в рамках проєктів $0116 U 003059$ та 0121U110543, а також проєкту Національного фонду досліджень України 2020.02/0089 (номер держреєстращї 0120U104004).

\section{ЦИТОВАНА ЛІТЕРАТУРА}

1. Boyko V.M., Popovych R.O., Shapoval N.M. Equivalence groupoids of classes of linear ordinary differential equations and their group classification.J. Phys. Conf. Ser. 2015. 621.012001. 17 pp. https://doi.org/10.1088/1742-6596/621/1/012002

2. Boyko V.M., Lokaziuk O.V., Popovych R.O. Admissible transformations and Lie symmetries of linear systems of second-order ordinary differential equations. 2021. arXiv:2105.05139. 
3. Wafo Soh C. Symmetry breaking of systems of linear second-order ordinary differential equations with constant coefficients. Commun. Nonlinear Sci. Numer. Simul. 2010. 15, № 2. P. 139-143. https://doi.org/10.1016/j.cnsns.2009.03.02

4. Meleshko S.V. Comment on "Symmetry breaking of systems of linear second-order ordinary differential equations with constant coefficients”. Commun. Nonlinear Sci. Numer. Simul. 2011. 16, № 9. P. 3447-3450. https://doi.org/10.1016/j.cnsns.2010.12.014

5. Campoamor-Stursberg R. Systems of second-order linear ODE's with constant coefficients and their symmetries. Commun. Nonlinear Sci. Numer. Simul. 2011. 16, № 8. P. 3015-3023. https://doi.org/10.1016/j.cnsns.2010.10.033

6. Campoamor-Stursberg R. Systems of second-order linear ODE's with constant coefficients and their symmetries. II. The case of non-diagonal coefficient matrices. Commun. Nonlinear Sci. Numer. Simul. 2012. 17, № 3. P. 1178-1193. https://doi.org/10.1016/j.cnsns.2011.08.002

7. Boyko V.M., Popovych R.O., Shapoval N.M. Lie symmetries of systems of second-order linear ordinary differential equations with constant coefficients. J. Math. Anal. Appl. 2013. 397, № 1. P. 434-440. https://doi.org/10.1016/j.jmaa.2012.06.030

8. Wafo Soh C., Mahomed F.M. Symmetry breaking for a system of two linear second-order ordinary differential equations. Nonlinear Dynam. 2000. 22, № 1. P. 121-133. https://doi.org/10.1023/A:1008390431287

9. Moyo S., Meleshko S.V., Oguis G.F. Complete group classification of systems of two linear second-order ordinary differential equations. Commun. Nonlinear Sci. Numer. Simul. 2013. 18, № 11. P. 2972-2983. https://doi.org/10.1016/j.cnsns.2013.04.012

10. Mkhize T.G., Moyo S., Meleshko S.V. Complete group classification of systems of two linear second-order ordinary differential equations: the algebraic approach. Math. Methods Appl. Sci. 2015. 38, № 9. P. 1824-1837. https://doi.org/10.1002/mma.3193

11. Suksern S., Moyo S., Meleshko S.V. Application of group analysis to classification of systems of three second-order ordinary differential equations. Math. Methods Appl. Sci. 2015. 38, № 18. P. 5097-5113. https://doi.org/10.1002/mma.3430

12. Gonzalez-Lopez A. Symmetries of linear systems of second-order ordinary differential equations. J. Math. Phys. 1988. 29, № 5. P. 1097-1105. https://doi.org/10.1063/1.527948

13. Winternitz P. Subalgebras of Lie algebras. Example of $\mathfrak{s l}(3, \mathbb{R})$. Symmetry in physics, CRM Proc. Lecture Notes. Vol. 34. Providence, RI: Amer. Math. Soc., 2004. P. 215-227.

Надійшло до редакції 30.06.2021

\section{REFERENCES}

1. Boyko, V. M., Popovych, R. O. \& Shapoval, N. M. (2015). Equivalence groupoids of classes of linear ordinary differential equations and their group classification. J. Phys. Conf. Ser., 621, 012001, 17 pp. https://doi.org/10.1088/1742-6596/621/1/012002

2. Boyko, V. M., Lokaziuk, O. V. \& Popovych, R. O. (2021). Admissible transformations and Lie symmetries of linear systems of second-order ordinary differential equations. arXiv:2105.05139.

3. Wafo Soh, C. (2010). Symmetry breaking of systems of linear second-order ordinary differential equations with constant coefficients. Commun. Nonlinear Sci. Numer. Simul., 15, No. 1, pp. 139-143. https://doi.org/10.1016/j.cnsns.2009.03.02

4. Meleshko, S. V. (2011). Comment on "Symmetry breaking of systems of linear second-order ordinary differential equations with constant coefficients". Commun. Nonlinear Sci. Numer. Simul., 16, No. 9, pp. 3447 3450. https://doi.org/10.1016/j.cnsns.2010.12.014

5. Campoamor-Stursberg, R. (2011). Systems of second-order linear ODE's with constant coefficients and their symmetries. Commun. Nonlinear Sci. Numer. Simul., 16, No. 8, pp. 3015-3023.

https://doi.org/10.1016/j.cnsns.2010.10.033

6. Campoamor-Stursberg, R. (2012). Systems of second-order linear ODE's with constant coefficients and their symmetries. II. The case of non-diagonal coefficient matrices. Commun. Nonlinear Sci. Numer. Simul., 17, No. 3, pp. 1178-1193. https://doi.org/10.1016/j.cnsns.2011.08.002 
7. Boyko, V. M., Popovych, R. O. \& Shapoval, N. M. (2013). Lie symmetries of systems of second-order linear ordinary differential equations with constant coefficients. J. Math. Anal. Appl., 397, No. 1, pp. 434-440. https://doi.org/10.1016/j.jmaa.2012.06.030

8. Wafo Soh, C. \& Mahomed, F. M. (2000). Symmetry breaking for a system of two linear second-order ordinary differential equations. Nonlinear Dynam., 22, No. 1, pp. 121-133. https://doi.org/10.1023/A:1008390431287

9. Moyo, S., Meleshko, S. V. \& Oguis, G. F. (2013). Complete group classification of systems of two linear second-order ordinary differential equations. Commun. Nonlinear Sci. Numer. Simul., 18, No. 11, pp. 29722983. https://doi.org/10.1016/j.cnsns.2013.04.012

10. Mkhize, T. G., Moyo, S. \& Meleshko, S. V. (2015). Complete group classification of systems of two linear second-order ordinary differential equations: the algebraic approach. Math. Methods Appl. Sci., 38, No. 9, pp. 1824-1837. https://doi.org/10.1002/mma.3193

11. Suksern, S., Moyo, S. \& Meleshko, S. V. (2015). Application of group analysis to classification of systems of three second-order ordinary differential equations. Math. Methods Appl. Sci., 38, No. 18, pp. 5097-5113. https://doi.org/10.1002/mma.3430

12. Gonzalez-Lopez, A. (1988). Symmetries of linear systems of second-order ordinary differential equations. J. Math. Phys., 29, No. 5, pp. 1097-1105. https://doi.org/10.1063/1.527948

13. Winternitz, P. (2004). Subalgebras of Lie algebras. Example of $\mathfrak{s l}(3, \mathbb{R})$. In Symmetry in physics (pp. 215227), CRM Proc. Lecture Notes, Vol. 34. Providence, RI: Amer. Math. Soc.

Received 30.06.2021

O.V. Lokaziuk, https://orcid.org/0000-0001-9663-251X

Institute of Mathematics of the NAS of Ukraine, Kyiv

E-mail: sasha.lokazuik@gmail.com

\section{LIE SYMMETRIES OF LINEAR SYSTEMS OF TWO SECOND-ORDER ORDINARY DIFFERENTIAL EQUATIONS}

We solve the complete group classification problem for the class of normal linear systems of second-order ordinary differential equations with two dependent variables over the real field. The proof essentially uses the description of admissible transformations of this class and Lie's theorem on realizations of Lie algebras on the line.

Keywords: linear systems of second-order ordinary differential equations, Lie symmetry, algebraic method of group classification, equivalence group. 